\title{
A comprehensive analysis of SVPWM for a Five- phase VSI based on SiC devices applied to motor drives.
}

\author{
Fernando Acosta-Cambranis \\ Department of Electronic Engineering \\ Universitat Politècnica de Catalunya \\ Terrassa, Spain \\ fernando.geovany.acosta.cambra@upc.edu
}

\author{
Jordi Zaragoza \\ Department of Electronic Engineering \\ Universitat Politècnica de Catalunya \\ Terrassa, Spain \\ jordi.zaragoza-bertomeu@upc.edu
}

\author{
Luis Romeral \\ Department of Electronic Engineering \\ Universitat Politècnica de Catalunya \\ Terrassa, Spain \\ luis.romeral@upc.edu
}

\begin{abstract}
This paper presents a comprehensive analysis of SVPWM for a five-phase VSI based on SiC devices applied to motor drives. The modulation techniques analyzed use medium and large vectors to reach the reference vector. The 2L SVPWM uses two large space vectors, and the generated output signal contain low frequency harmonics. $2 L+2 M$ SVPWM uses two large and two medium space vectors. This technique provides good power loss distribution. 4L SVPWM works with the activation of four large space vectors. This modulation is able to generate low common-mode voltage. The performance and main features are analyzed using Matlab/Simulink and PLECS blockset software. Power losses, total harmonic distortion and common-mode voltage are compared and evaluated.
\end{abstract}

Keywords-Five-phase inverter, SVPWM, Total harmonic distortion, power losses, Common-mode Voltage, Silicon Carbide.

\section{INTRODUCTION}

Multi-phase machines are used in many applications, such as power generation, electric propulsion systems and power industrial applications [1]-[3]. Some of the advantages of the multi-phase machines are: the reductions in the amplitude of the torque pulsation, improves the torque per ampere, more degrees of freedom, better fault tolerance, improves the sharing of the power between the inverter phase legs, and higher reliability, among others [2], [4]-[6]. These machines are normally supplied from two-level voltage source inverters (VSI).

Carrier-based PWM (CBPWM) and space vector PWM (SVPWM) are the most used modulation techniques for the multi-phase inverter [2], [7]-[9]. Thus, each technique demostrates different performance and advantages over each other, in terms of power losses distribution, commonmode voltage (CMV) [10]-[12], the harmnic content, and the shapes of the output voltage and current signal.

Efficiency in electric systems plays an important role in the design and implementation of electric inverters. Having a system with high efficiency depends on reducing the losses in the power semiconductor devices of the inverter. The power losses in the power semiconductor devices are divided into conduction and switching losses. Conduction losses result from the current passing through every device and the switching losses depends on the switching frequency [13]-[15].

Using wide bandgap (WBG) power devices allows the inverter to work with higher switching frequencies, while operating at high voltages and high-temperatures. One wide bandgap device is based on silicon carbide $(\mathrm{SiC})$.

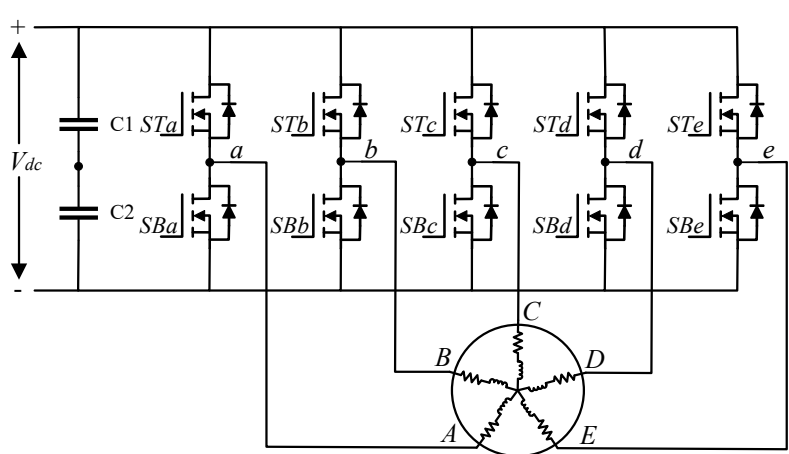

Fig. 1. Five-phase inverter

These devices perform better than the silicon ( $\mathrm{Si}$ ) devices, due to the low on-resistance $R_{o n}$, thus resulting in power devices with better performance, also are able to operate with higher breakdown voltage, higher temperatures of up to $600^{\circ} \mathrm{C}$, but actually the industrial $\mathrm{SiC}$ devices can operate up to $175^{\circ} \mathrm{C}$ this due to that there are no high temperature packaging techniques [16], [17].

The objective of this work unlike others papers is to analize the power losses, quantify CMV and study the harmonic content of a five-phase VSI based on SiC applied to an electric traction system under different modulation techniques. In order to analyze the converter a model based on Matlab Simulink/Plecs software has been developed. Fig. 1 shows the topology of the implemented two-level five-phase inverter with $\mathrm{SiC}$ power devices applied to a Permanente Magnet assisted Synchronous Reluctance Machine (PMa-SynRM). Fig. 2 shows the PWM techniques implemented in this paper: the Two Large Vectors (2L PWM) [2], [8], [18]-[20], Two Large Two Medium Vectors (2L+2M PWM) [2], [7], [8], [18], [20]-[22] and Four Large Vectors (4L PWM) [21], [23].

\section{FIVE-PHASE VSI}

In the multi-phase inverter, the number of states can be calculated by $2^{n}$ (where $n$ is equal to the number of phases).

The five-phase inverter has 32 switching states, where two of these states are null or zero states. The switching states can be read like binary numbers with five digits where " 1 " means that the upper semiconductor device is closed and the lower device is open. The " 0 " state means that the lower device is closed and the upper device is open. Phase $a$ is the most significant bit, and phase $e$ is the least significant bit[4], [8], [24].

The instantaneous phase voltages of each leg are expressed as follows[2], [18]: 


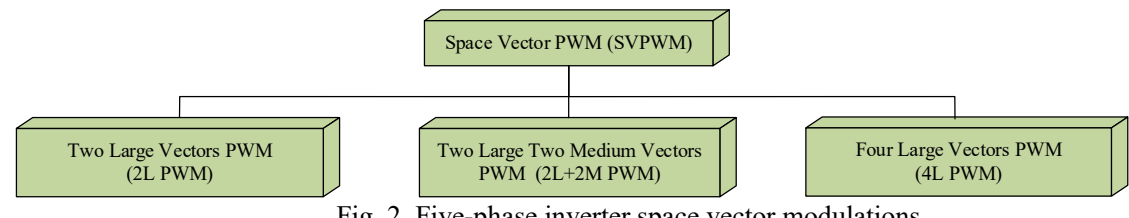

Fig. 2. Five-phase inverter space vector modulations.

$$
\begin{aligned}
V_{a} & =\frac{V_{d c}}{2}\left(\frac{4}{5} S_{a}-\frac{1}{5}\left[S_{b}+S_{c}+S_{d}+S_{e}\right]\right) \\
V_{b} & =\frac{V_{d c}}{2}\left(\frac{4}{5} S_{b}-\frac{1}{5}\left[S_{a}+S_{c}+S_{d}+S_{e}\right]\right) \\
V_{c} & =\frac{V_{d c}}{2}\left(\frac{4}{5} S_{c^{-}} \frac{1}{5}\left[S_{a}+S_{b}+S_{d}+S_{e}\right]\right) \\
V_{d} & =\frac{V_{d c}}{2}\left(\frac{4}{5} S_{d^{-}} \frac{1}{5}\left[S_{a}+S_{b}+S_{c}+S_{e}\right]\right) \\
V_{e} & =\frac{V_{d c}}{2}\left(\frac{4}{5} S_{e^{-}} \frac{1}{5}\left[S_{a}+S_{b}+S_{c}+S_{d}\right]\right)
\end{aligned}
$$

where $S_{i}(i=a, b, c, d, e)$ is the switching state of each one of the legs, $V_{i}$ the instantaneous phase voltage, and $V_{d c}$ the dc bus voltage.

\section{SPACE Vector Modulation (SVPWM)}

The five-phase VSI has 32 switching states (i.e. vectors) for wich 30 are active vectors and 2 are zero vectors. An nphase system corresponds to an $n$ - 1 dimensional space ( $n$ it is assumed is odd, the load is balanced and star-connected). The 4-dimensional space where the vectors are projected can be divided into 2D subspaces by mean of decoupling transformations. In this work, the Clarke Transformation $\left(C_{T}\right)$ is applied as follows:

$$
C_{T}=\frac{2}{5}\left[\begin{array}{ccccc}
1 & \cos (\varphi) & \cos (2 \varphi) & \cos (3 \varphi) & \cos (4 \varphi) \\
0 & \sin (\varphi) & \sin (2 \varphi) & \sin (3 \varphi) & \sin (4 \varphi) \\
1 & \cos (2 \varphi) & \cos (4 \varphi) & \cos (\varphi) & \cos (3 \varphi) \\
0 & \sin (2 \varphi) & \sin (4 \varphi) & \sin (\varphi) & \sin (3 \varphi) \\
1 / 2 & 1 / 2 & 1 / 2 & 1 / 2 & 1 / 2
\end{array}\right]
$$

where $\varphi=2 \pi / 5$ for the case of the five-phase VSI.

$$
\left[\begin{array}{l}
v_{d 1} \\
v_{q 1} \\
v_{d 2} \\
v_{q 2} \\
v_{\mathrm{z}}
\end{array}\right]=C_{T} *\left[\begin{array}{l}
v_{a} \\
v_{b} \\
v_{c} \\
v_{d} \\
v_{e}
\end{array}\right]
$$

By applying (2) to the five sinusoidal references, we can obtain two $2 D$ subspaces with ten sectors for each one of them. Fig. 3 shows the first subspace $d_{1}-q_{1}$, which contains the fundamental component and the $10 k \pm 1(k=0,1,2, \ldots)$ harmonics. The second subspace $d_{2}-q_{2}$, contains the harmonics on the order of $10 k \pm 3$. The $5 k$ harmonics cannot exist in this case because of the star configuration of the load.

The subspace $d_{l}-q_{l}$ contains four-types of space vectors of different sizes. In Fig. 3, the small (green) and large (blue) vectors are formed by the combination of three legs of the VSI in the turn-on state and two legs in turn-off (11100), or vice verse (00011). Medium (red) vectors are formed by four legs turned-on and one turned-off (11110) or vice verse $(01111)$. The zero vectors are all the legs turned-on (11111) or turned-off (00000).
In applying (2), the signal reference is transformed into a rotary vector (Vref) positioned in the $d_{l}-q_{l}$ subspace. To apply the different SVPWM techniques, it is necessary to calculate the magnitude and position of the reference vector as follows:

$$
\begin{gathered}
V_{r e f}=\sqrt{V_{d_{1}}{ }^{2}+V_{q_{1}}{ }^{2}} \\
\alpha=\tan ^{-1}\left(\frac{V_{d_{1}}}{V_{q_{1}}}\right)
\end{gathered}
$$

With the position and magnitude of the reference vector obtained from (4) and (5), the activation time of each switch combination can be calculated in order to reach the reference vector.

\section{A. Two Large Vectors (2L SVPWM)}

2L SVPWM use two large vectors plus the zero vectors to reach the reference vector. Applying 2L SVPWM, the inverter can supply more current than the other modulation techniques. This is able to maximize the modulation index $(m=1.2311)$.

The activation sequence of the space vectors in the $2 \mathrm{~L}$ SVPWM technique depends on wheter the location of the reference vector is an odd or an even sector.

Fig. 4 (b) shows the switching sequence for an odd sector that is $V_{0}, V_{B}, V_{A}, V_{31}, V_{A} V_{B}, V_{0}$. For an even sector the switching sequence is $V_{0}, V_{A}, V_{B}, V_{31}, V_{B} V_{A}, V_{0}$.

\section{B. Two Large Two Medium Vectors $(2 L+2 M S V P W M)$}

Unlike the 2L SVPWM modulation, the $2 \mathrm{~L}+2 \mathrm{M}$ SVPWM combines two large and two medium vectors plus the zero vectors. This modulation has a maximum modulation index of $m=1.0515$. This modulation technique eliminates the low frequency harmonics in the voltages and the currents of the inverter[8].

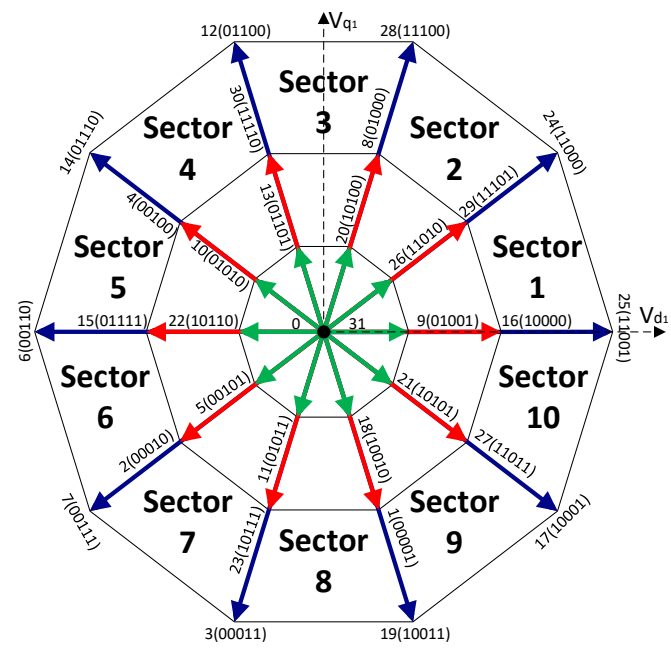

Fig. 3. 2D subspace vectors $d_{1}-q_{1}$. 
Fig. 5 (b) shows the switching sequence for an odd sector $V_{0}, V_{A M}, V_{B L}, V_{A L}, V_{B M}, V_{31}, V_{B M}, V_{A L}, V_{B L}, V_{A M}, V_{0}$. For an even sector, the switching sequence is $V_{0}, V_{B M}, V_{A L}, V_{B L}$, $V_{A M}, V_{31}, V_{A M}, V_{B L}, V_{A L}, V_{B M}, V_{0}$.

\section{Four Large Vectors PWM (4L SVPWM)}

The $4 \mathrm{~L}$ PWM like the $2 \mathrm{~L}+2 \mathrm{M}$ PWM, is formed by four active vectors plus two zero vectors, but the difference is that it has only large vectors. This technique uses the two vectors of the sector where the reference vector is located and the large vector from the previous and next sector. $4 \mathrm{~L}$ PWM modulation has a modulation index of $m=1.0515$.

Fig. 6 (b) shows the switching sequence for an odd sector that is $V_{0}, V_{C L}, V_{A L}, V_{B L}, V_{D L}, V_{31}, V_{D L}, V_{B L}, V_{A L}, V_{C L}$, $V_{0}$. For an even sector the switching sequence is $V_{0}, V_{D L}$, $V_{B L}, V_{A L}, V_{C L}, V_{31}, V_{C L}, V_{A L}, V_{B L}, V_{D L}, V_{0}$.

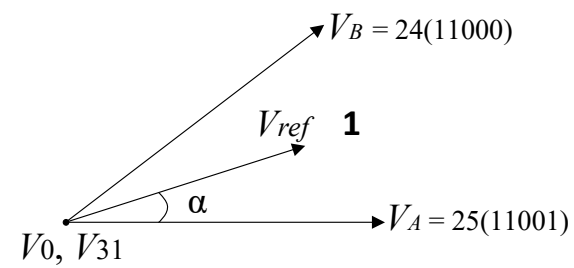

(a)

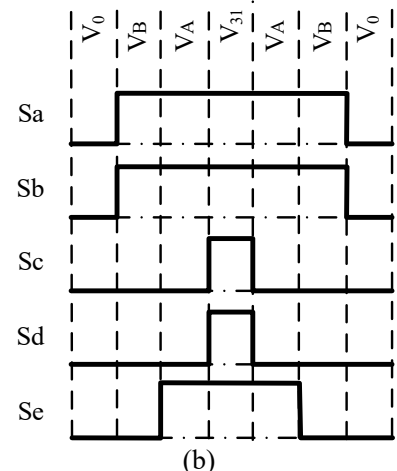

Fig. 4. 2L SVPWM Sector 1: (a) space vectors and (b) switching sequence.

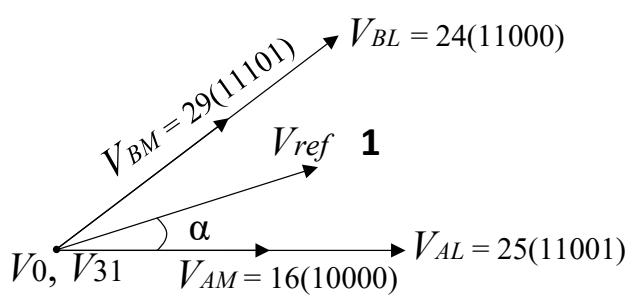

(a)

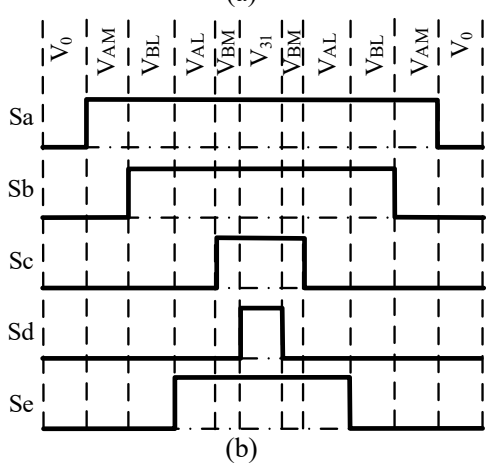

Fig. 5. 2L +2M SVPWM Sector 1: (a) space vectors and (b) switching sequence.

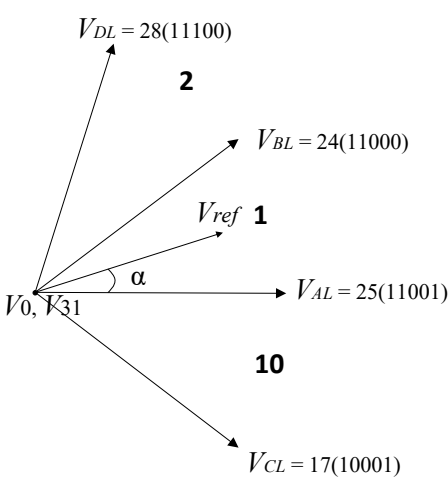

(a)

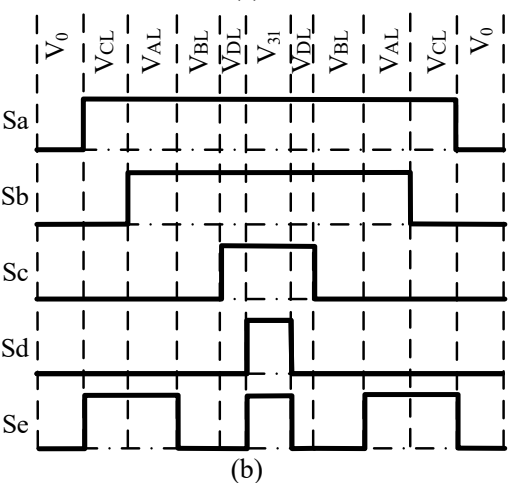

Fig. 6. 4L SVPWM Sector 1: (a) space vectors and (b) switching sequence.

\section{SIMULATION RESULTS}

In order to study the different modulation techniques and compare them, the following characteristics have been evaluated: A) the harmonic content, B) the CMV generated and $\mathrm{C}$ ) the power losses.

\section{A. Harmonic content}

The output voltages and currents are evaluated using the total harmonic distortion (THD) and the weighted total harmonic distortion (WTHD). The parameters are defined as follows:

$$
\begin{gathered}
\operatorname{THD}(\%)=100 \sqrt{\frac{\sum_{n=2}^{\infty} V_{n, R M S}^{2}}{V_{1, R M S}^{2}}}=100 \sqrt{\left(\frac{V_{R M S}}{V_{1, R M S}}\right)^{2}-1} \\
\operatorname{WTHD}(\%)=100 \sqrt{\frac{\sum_{n=2}^{\infty}\left(\mathrm{V}_{\mathrm{n}, \mathrm{RMS} / \mathrm{n})^{2}}\right.}{V_{1, R M S}^{2}}}
\end{gathered}
$$

\section{B. Power Losses}

\section{1) Conduction losses}

Conduction occurs when the MOSFET is in the onstate. These losses are defined in the form:

$$
P_{\text {condloss }}=\frac{1}{T_{s}} \int_{0}^{T_{s}}\left(V_{F 0}+R_{o n} I_{F}\right) * I_{F} d t
$$

Where $V_{F 0}$ is the threshold voltage, $R_{o n}$ is the on resistance, $T_{s}$ is the period of the fundamental frequency and $I_{F}$ is the forward current.

\section{2) Switching losses}

These losses are generated during the turn-on and turnoff switching processes of the power devices, and they are defined in the following form: 


$$
P_{\text {Swtoss }}=\frac{1}{T_{s}} \sum_{j=1}^{n T_{s}}\left[E_{o n_{j}}\left(I_{F}, V_{\text {offs }}\right)+E_{o f f}\left(I_{F}, V_{o f f s}\right)\right]
$$

where $E_{\text {on }}$ and $E_{\text {off }}$ are the turn-on and turn-off energy dissipated during the change of state, $n T_{s}$ is the number of transitions in one fundamental period, $I_{F}$ is the on-state current running through the power devices and $V_{\text {offs }}$ is the voltage in the off-state.

\section{3) Efficiency}

Efficiency is defined in the following form:

$$
\eta(\%)=\left(\frac{P_{\text {tot }}-P_{\text {condloss }}-P_{\text {swtloss }}}{P_{\text {tot }}}\right) * 100
$$

where $P_{t o t}$ is the inverter total power.

\section{Common-Mode Voltage (CMV)}

The CMV in motor applications can cause bearing currents that lead to bearing failures, electromagnetic interference (EMI), damage to the winding insulation, and mechanical vibration. Table I shows the CMV generated for each of the different space vectors [11], [25].

TABLE I. CMV FOR EACH VECTOR TYPE.

\begin{tabular}{|c|c|}
\hline Vector type & Common-Mode Voltage \\
\hline Zero & $0.5^{*} V_{d c}$ \\
\hline Small & $0.1^{*} V_{d c}$ \\
\hline Medium & $0.3^{*} V_{d c}$ \\
\hline Large & $0.1^{*} V_{d c}$ \\
\hline
\end{tabular}

Applying the Fast Fourier Transform (FFT) to the CMV signal, and the sum of the all the components of the FFT spectrum squared and divided between the half of the dc bus $V_{d c}$, we can obtain the normalized energy analysis of the CMV. The above mentioned is represented as follows:

$$
E_{\text {norm }} \approx \sum_{i=1}^{\infty}\left[\frac{x(i)}{\left(\mathrm{V}_{\mathrm{dc}} / 2\right)}\right]^{2}
$$

where $E_{\text {norm }}$ is the normalized energy of the CMV, $i$ the number of elements of the FFT spectrum and $x$ the FFT spectrum of the CMV.

\section{Simulation}

All the modulation techniques and the five-phase VSI are implemented in Matlab/Simulink and PLECS blockset software. Fig.7 (a) and (b) shows the switching energy and conduction losses of the SiC MOSFET C2M0160120D used in this analysis. Table II shows the simulation parameters of the system.

TABLE II. SimUlation PARAMETERS

\begin{tabular}{|c|c|}
\hline Inverter Switching frequency $(f s)$ & $100 \mathrm{kHz}$ \\
\hline Inverter dc bus link $\left(V_{d c}\right)$ & $550 \mathrm{~V}$ \\
\hline Inverter temperature $(T)$ & $125^{\circ} \mathrm{C}$ \\
\hline Motor Stator Resistance & $2.05 \Omega$ \\
\hline Motor Stator Inductance & $59.89 \mathrm{mH}$ \\
\hline Motor Nominal Power & $3 \mathrm{~kW}$ \\
\hline Motor Nominal Torque & $7 \mathrm{Nm}$ \\
\hline Motor Nominal Speed & $4000 \mathrm{rpm}$ \\
\hline Motor Pair of poles & 6 \\
\hline
\end{tabular}

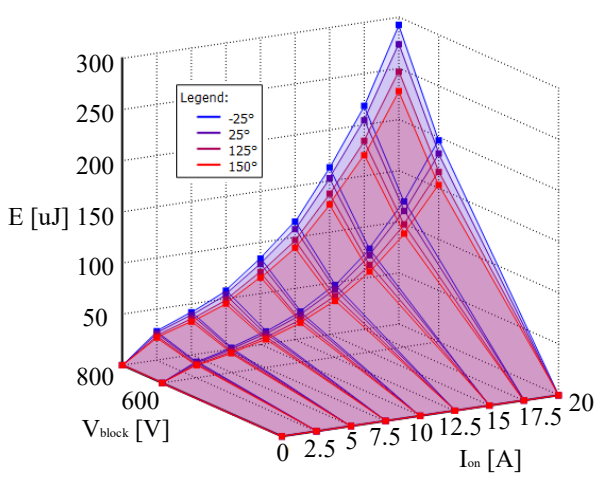

(a)

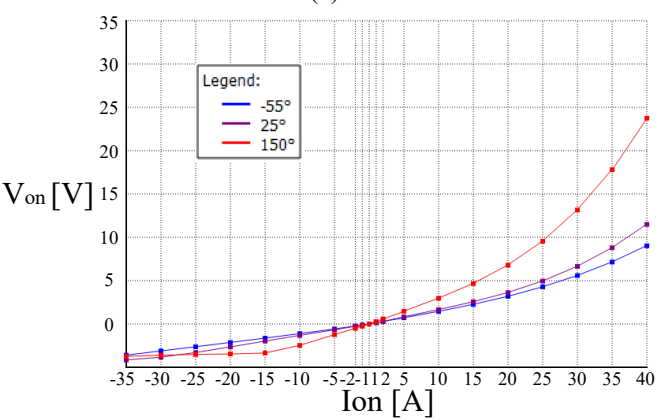

(b)

Fig. 7. PLECS lookup tables for the MOSFET C2M0160120D: (a) Switching and (b) conduction losses.

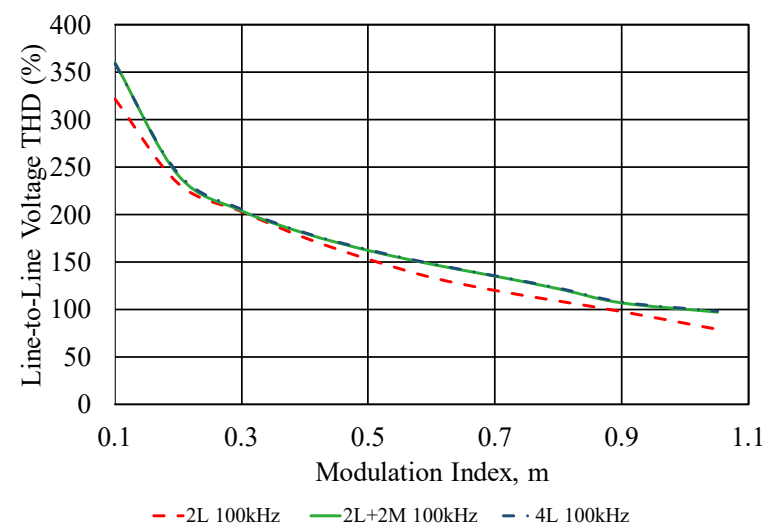

(a)

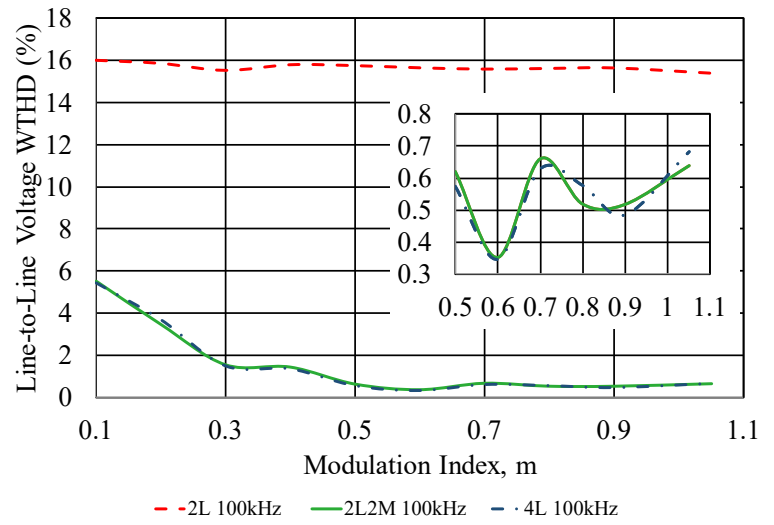

(b)

Fig. 8. Line-to-line voltage harmonic distortion: (a) THD and (b) WTHD.

Fig. 8 (a) and (b) show the THD and WTHD for the first two thousand harmonics of the line-to-line voltage. The $2 \mathrm{~L}$ SVPWM is 9 to $38 \%$ smaller compared with the other two modulations. However, the WTHD for the 2L SVPWM as 
much as to 10 to $15 \%$ greater than the $2 \mathrm{~L}+2 \mathrm{M}$ and $4 \mathrm{~L}$ SVPWM, due to the presence of low order harmonics in the 2L SVPWM.

Fig. 9 (a) and (b) show the THD and WTHD of the current output for $2 \mathrm{~L}, 2 \mathrm{~L}+2 \mathrm{M}$ and $4 \mathrm{~L}$ SVPWM. The $2 \mathrm{~L}+2 \mathrm{M}$ and $4 \mathrm{~L}$ SVPWM have similar values with a slight difference. However, compared with the 2L SVPWM that has a 6 to $10 \%$ THD greater and a 2.5 to $3 \%$ greater WTHD, we can see that these results are also due to the presence of low order harmonics.

In Fig. 10, can be observed the CMV energy comparison. 4L SVPWM has the lowest energy and this decreases according to the increase in the modulation index. The energy decreases in the three different modulations because the activation time of the zero vectors is reduced when the modulation index increases. Table III shows the maximum reduction in the $\mathrm{CMV}$ of the modulations.

TABLE III. CMV REDUCTION PERCENTAGE.

\begin{tabular}{|c|c|}
\hline 2L SVPWM & $60-80 \%$ \\
\hline 2L+2M SVPWM & $62 \%$ \\
\hline 4L SVPWM & $80 \%$ \\
\hline
\end{tabular}

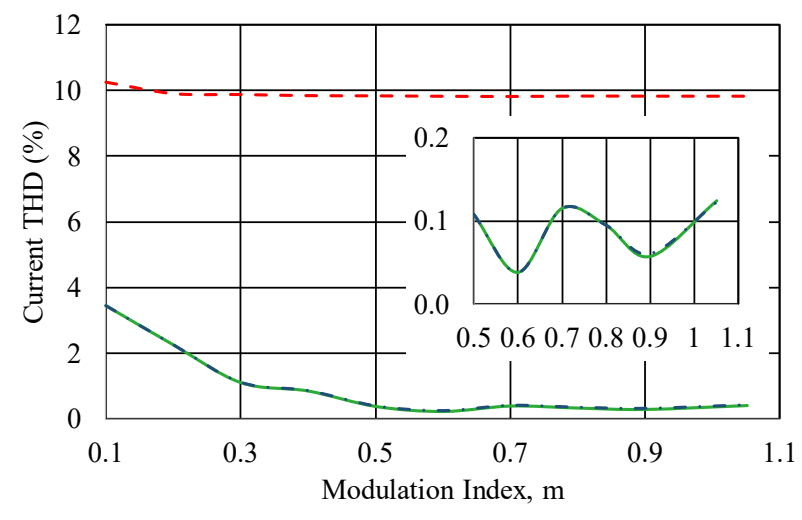

$--2 \mathrm{~L} 100 \mathrm{kHz} \quad-2 \mathrm{~L} 2 \mathrm{M} 100 \mathrm{kHz} \quad-\cdot 4 \mathrm{~L} 100 \mathrm{kHz}$

(a)

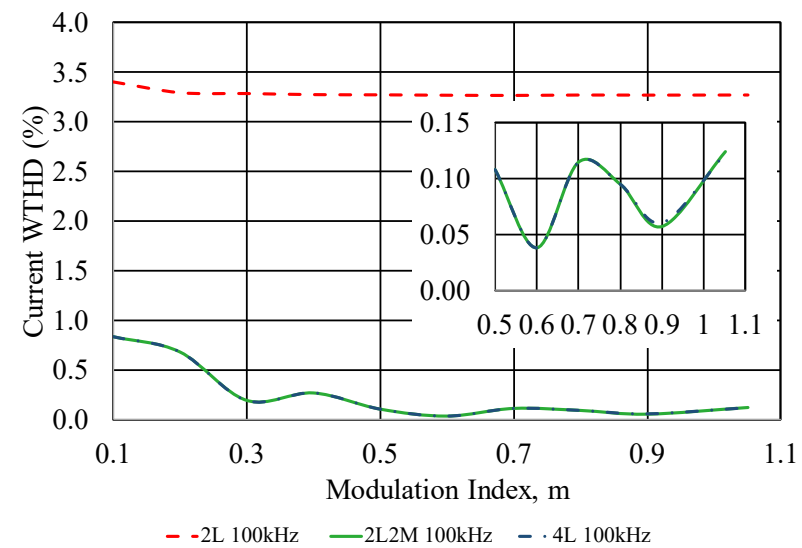

(b)

Fig. 9. Current harmonic distortion: (a) THD and (b) WTHD.

Fig. 11 (a), provides the efficiency comparison of the modulations, according to the change in motor speed using the nominal torque. The 4L SVPWM has the lowest efficiency. Meanwhile the 2L and 2L+2M SVPWM have a similar efficiency.

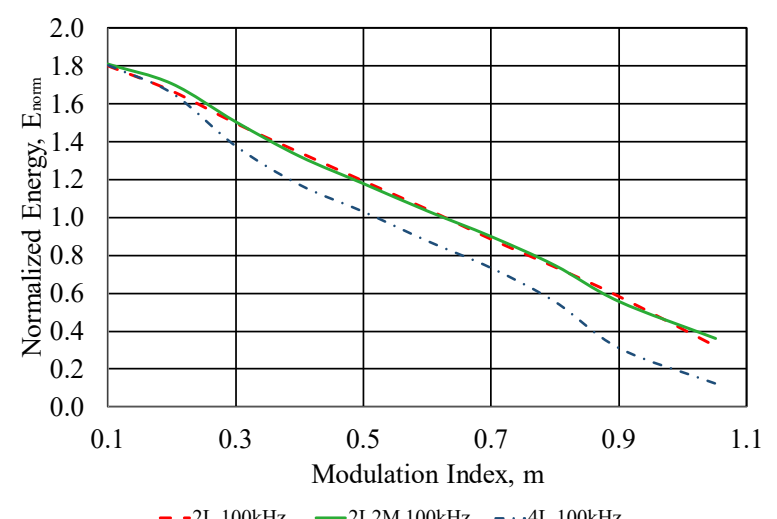

$--2 \mathrm{~L} 100 \mathrm{kHz} \quad-2 \mathrm{~L} 2 \mathrm{M} 100 \mathrm{kHz} \quad-\cdots 4 \mathrm{~L} 100 \mathrm{kHz}$

Fig. 10. Energy analysis of the FFT CMV.

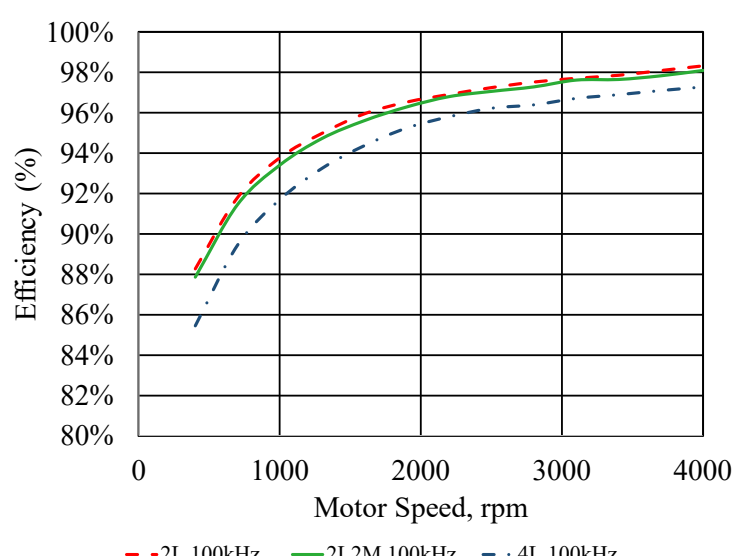

(a)

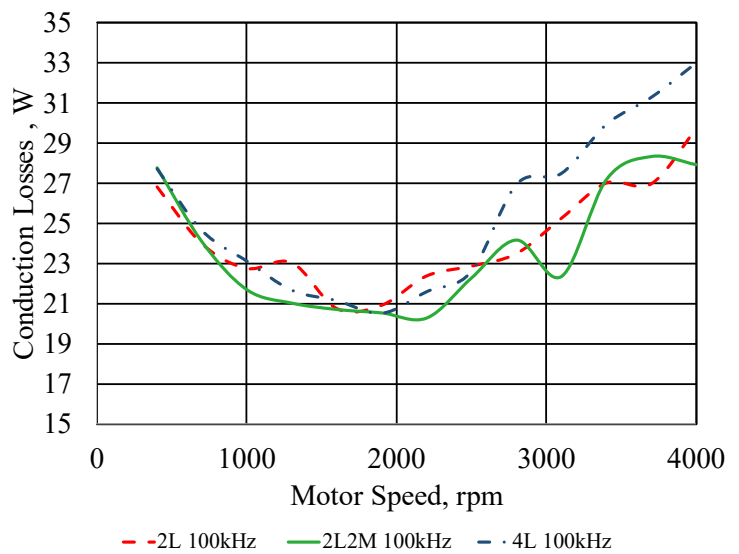

(b)

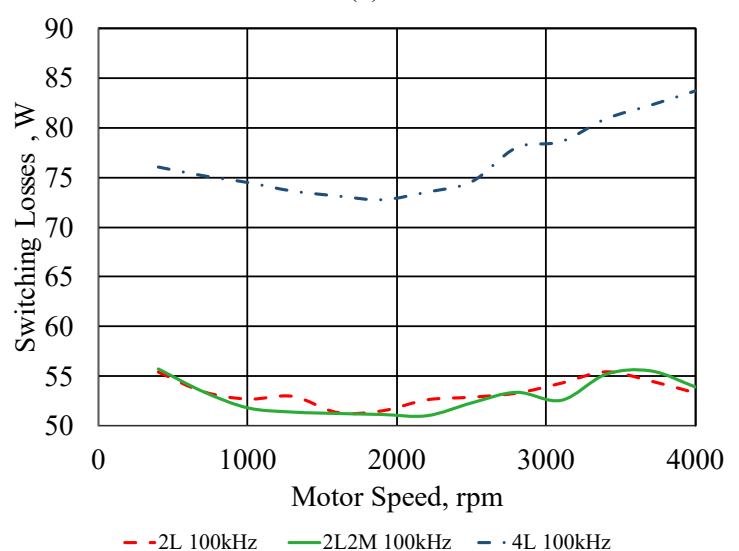

(c)

Fig. 11 Modulations comparison on: (a) Efficiency, (b) Conduction Losses and (c) Switching Losses. 
Fig. 11 (b) shows the conduction losses of the modulations. These losses are similar but the 4L SVPWM can have up to 6 to $18 \%$ more losses than the other modulations.

In the Fig. 11 (c), can be observed the switching losses. The main reason that the 4L SVPWM has the lowest efficiency is due to the switching losses. The 4L SVPWM has between 36 to $55 \%$ more losses than the other modulations. On the other hand, the $2 \mathrm{~L}$ and $2 \mathrm{~L}+2 \mathrm{M}$ have similar losses, and depending on the operating zone, one can have a few more losses using one over the other.

\section{CONCLUSIONS}

Depending on the application of the multiphase machine or the characteristic that one wants to improve, each of these modulation techniques has advantages and disadvantages. The $4 \mathrm{~L}$ SVPWM allows us to reduce the $\mathrm{CMV}$, and thus reduce the bearing currents in the multiphase machine, although the efficiency of the inverter becomes compromised. The $2 \mathrm{~L}+2 \mathrm{M}$ modulation has good WTHD content. We therefore have a good current waveform and lower power losses, but the reduction in CMV is lower than that of $4 \mathrm{~L}$ SVPWM. Finally, the $2 \mathrm{~L}$ SVPWM can provide almost the same reduction in CMV while at the same time it has less losses than the $4 \mathrm{~L}$ SVPWM, but with the disadvantage that its current THD and WTHD is higher due to the presence of low order harmonics.

\section{ACKNOWLEDGMENT}

This work was supported by the Ministerio de Ciencia, Innovación y Universidades under Project CICYT (TRA2016-80472-R), the Consejo Nacional de Ciencia y Tecnología (CONACYT) of México (scholarship number 496458) and Secretaria d'Universitats i Recerca del Departament d'Empresa i Coneixement de la Generalitat de Catalunya.

\section{REFERENCES}

[1] E. Levi, "Multiphase Electric Machines for Variable-Speed Applications," IEEE Trans. Ind. Electron., vol. 55, no. 5, pp. 18931909, May 2008.

[2] K. A. Chinmaya and M. Udaya Bhasker, "Analysis of different space vector pulse width modulation techniques for five-phase inverters," 11th IEEE India Conf. Emerg. Trends Innov. Technol. INDICON 2014, pp. 1-6, 2014.

[3] I. Lopez et al., "Generalized PWM-Based Method for Multiphase Neutral-Point-Clamped Converters With Capacitor Voltage Balance Capability," IEEE Trans. Power Electron., vol. 32, no. 6, pp. 48784890, Jun. 2017.

[4] W. N. Wan Abdul Munim, M. F. Ismail, A. F. Abidin, and H. Che Mat Haris, "Switching technique comparison for multi-phase inverters," in 2013 IEEE 7th International Power Engineering and Optimization Conference (PEOCO), 2013, no. June, pp. 155-160.

[5] G. . Singh, "Multi-phase induction machine drive research-a survey," Electr. Power Syst. Res., vol. 61, no. 2, pp. 139-147, Mar. 2002.

[6] F. Barrero and M. J. Duran, "Recent Advances in the Design, Modeling, and Control of Multiphase Machines-Part I," IEEE Trans. Ind. Electron., vol. 63, no. 1, pp. 449-458, Jan. 2016.

[7] S. C. Rangari, "Implementation of Large and Medium Vectors for SVPWM technique in five phase Voltage Source Inverter," in International Conference on Intelligent Computing and Control Systems (ICICCS), 2017, no. 1, pp. 751-756.
[8] W. N. Wan Abdul Munim, M. F. Ismail, A. F. Abidin, and H. Che Mat Haris, "Multi-phase inverter Space Vector Modulation," in 2013 IEEE 7th International Power Engineering and Optimization Conference (PEOCO), 2013, no. June, pp. 149-154.

[9] A. Iqbal and S. Moinuddin, "Comprehensive Relationship Between Carrier-Based PWM and Space Vector PWM in a Five-Phase VSI," IEEE Trans. Power Electron., vol. 24, no. 10, pp. 2379-2390, Oct. 2009 .

[10] X. Wu, G. Tan, Z. Ye, Y. Liu, and S. Xu, "Optimized CommonMode Voltage Reduction PWM for Three-Phase Voltage-Source Inverters," IEEE Trans. Power Electron., vol. 31, no. 4, pp. 29592969, Apr. 2016.

[11] M. J. Durán, J. Prieto, and F. Barrero, "Space Vector PWM With Reduced Common-Mode Voltage for Five-Phase Induction Motor Drives Operating in Overmodulation Zone," IEEE Trans. Power Electron., vol. 28, no. 8, pp. 4030-4040, Aug. 2013.

[12] O. Lopez et al., "Space-Vector PWM With Common-Mode Voltage Elimination for Multiphase Drives," IEEE Trans. Power Electron., vol. 31, no. 12, pp. 8151-8161, Dec. 2016.

[13] K. Berringer, J. Marvin, and P. Perruchoud, "Semiconductor power losses in AC inverters,' in IAS '95. Conference Record of the 1995 IEEE Industry Applications Conference Thirtieth IAS Annual Meeting, 1995, vol. 1, pp. 882-888.

[14] S. Ceballos et al., "Efficient Modulation Technique for a Four-Leg Fault-Tolerant Neutral-Point-Clamped Inverter," IEEE Trans. Ind. Electron., vol. 55, no. 3, pp. 1067-1074, Mar. 2008.

[15] J. Pou, D. Osorno, J. Zaragoza, C. Jaen, and S. Ceballos, "Power losses calculation methodology to evaluate inverter efficiency in electrical vehicles," in 2011 7th International Conference-Workshop Compatibility and Power Electronics (CPE), 2011, pp. 404- 409.

[16] J. Millan, P. Godignon, X. Perpina, A. Perez-Tomas, and J. Rebollo, "A Survey of Wide Bandgap Power Semiconductor Devices," IEEE Trans. Power Electron., vol. 29, no. 5, pp. 2155-2163, May 2014.

[17] B. Ozpineci, L. M. Tolbert, S. K. Islam, and M. Hasanuzzaman, "Effects of silicon carbide (SiC) power devices on HEV PWM inverter losses," in IECON'01. 27th Annual Conference of the IEEE Industrial Electronics Society (Cat. No.37243), 2002, vol. 2, no. C, pp. 1061-1066.

[18] D. Dujic, M. Jones, and E. Levi, "Continuous Carrier-Based vs. Space Vector PWM for Five-Phase VSI," in EUROCON 2007 - The International Conference on "Computer as a Tool," 2007, pp. 17721779 .

[19] M. S. Bin Zulkifli, W. N. B. W. A. Munim, and H. C. M. Haris, "Five Phase Space Vector Modulation Voltage Source Inverter using large vector only," in 2012 International Symposium on Computer Applications and Industrial Electronics (ISCAIE), 2012, no. Iscaie, pp. 5-9.

[20] A. Iqbal and E. Levi, "Space vector modulation schemes for a fivephase voltage source inverter," in 2005 European Conference on Power Electronics and Applications, 2005, pp. 12 pp.-P.12.

[21] J. Prieto, M. Jones, F. Barrero, E. Levi, and S. Toral, "Comparative Analysis of Discontinuous and Continuous PWM Techniques in VSI-Fed Five-Phase Induction Motor," IEEE Trans. Ind. Electron., vol. 58, no. 12, pp. 5324-5335, Dec. 2011.

[22] Shan Xue and Xuhui Wen, "Simulation Analysis of Two Novel Multiphase SVPWM Strategies," in 2005 IEEE International Conference on Industrial Technology, 2005, pp. 1337-1342.

[23] J. Prieto, F. Barrero, S. Toral, E. Levi, M. Jones, and M. J. Durán, "Analytical Evaluation of Switching Characteristics in Five-Phase Drives with Discontinuous Space Vector Pulse Width Modulation Techniques," EPE J., vol. 23, no. 2, pp. 24-33, Jun. 2013.

[24] R. Shi and H. A. Toliyat, "Vector control of five-phase synchronous reluctance motor with space vector pulse width modulation (SVPWM) for minimum switching losses," in APEC. Seventeenth Annual IEEE Applied Power Electronics Conference and Exposition (Cat. No.02CH37335), 2002, vol. 00, no. c, pp. 57-63.

[25] S. M. Dabour, A. S. Abdel-Khalik, A. M. Massoud, and S. Ahmed, "Analysis of Scalar PWM Approach with Optimal Common-Mode Voltage Reduction Technique for Five-Phase Inverters," IEEE J. Emerg. Sel. Top. Power Electron., vol. PP, no. c, pp. 1-1, 2018. 